\title{
Maquetes e mapas de fluxo: ferramentas para o design da sinalização do Centro Universitário Norte do Espírito Santo (Ceunes-Ufes) Models and Flow maps: Tools to Design Centro Universitário Norte do Espírito Santo (Ceues-Ufes) Signage
}

\author{
Ricardo Esteves, Mauro Pinheiro, Viviani Carnielli
}

wayfinding, sinalização, maquetes, mapas, metodologia

O artigo trata do desenvolvimento do projeto de sinalização para o Centro Universitário Norte do Espírito Santo (Ceunes-Ufes), localizado na Cidade de São Mateus (ES). O projeto foi desenvolvido pelo laboratório de projetos em design da Universidade Federal do Espírito Santo, localizado em Vitória (ES). São descritas as etapas de levantamento e sistematização de dados, destacando-se as estratégias de comunicação que orientaram a seleção das informações a serem dispostas na sinalização. Destacam-se as ferramentas e técnicas de validação criadas para avaliar a pertinência do plano de sinalização proposto, a saber: maquetes e mapas de fluxo. Conclui que essas ferramentas foram fundamentais no processo de validação do plano de sinalização, considerando que a distância de $214 \mathrm{~km}$ entre o Centro Universitário e o laboratório onde se encontrava a equipe envolvida no projeto dificultava a realização de visitas para conferir as informações in loco.

wayfinding, signage, models, maps, methodology

This article looks at the development of the signage system for Centro Universitário Norte do Espírito Santo (Ceunes-Ufes), located in the city of Sao Mateus, ES, Brazil. The project has been developed by a design lab from the design department of Universidade Federal do Espírito Santo, located in Vitória, ES. The data searching and organizing phases are described in this article as well as the communication strategies that guided the definition of the information to be displayed in the signage system. The tools and the validation techniques created to evaluate the proposed signage plan are models and flow maps. The outcome is that these tools were fundamental in the process of validating the signage plan. It is important to consider the distance of 133 miles between campus and the lab where the project staff was located, making it difficult for the group to travel to check information on site.

\section{Introdução}

Projetos de sinalização ${ }^{1}$ costumam lidar com questões que se relacionam a diferentes áreas de conhecimento. De acordo com Velho (2007), um projeto de sinalização pode ser caracterizado pelo resultado da combinação de vários subsistemas como: informações; gráfico; físico/formal;

\footnotetext{
${ }^{1} \mathrm{Na}$ literatura encontram-se diversas denominações, com sentidos ligeiramente diferentes, que buscam definir projetos de sinalização. A discussão sobre os diferentes sentidos que esses termos apresentam foge ao escopo deste artigo para aprofundar-se nessa questão sugerimos a leitura de ADG Brasil (2004), CALORI (2007), COSTA (2007), PASSINI e ARTHUR (2002), FOLLIS e HAMMER (1979), VELHO (2007). Aqui o sentido de "projeto de sinalização" refere-se ao planejamento, projeto e especificação de elementos gráficos no ambiente construído ou natural. Estes elementos são usados para comunicar informações específicas em sistemas de identificação, informação, direção, interpretação, orientação, regulamentação e ambientação.
}

R. Esteves; M. Pinheiro \& V. Carnielli. 2015. Maquetes e mapas de fluxo: ferramentas para o design da sinalização do Centro Universitário Norte do Espírito Santo (Ceunes-Ufes). In: C. G. Spinillo; L. M. Fadel; V. T. Souto; T. B. P. Silva \& R. J. Camara (Eds) Anais [Pôster] do $7^{\circ}$ Congresso Internacional de Design da Informação/Proceedings [Poster] of the 7th Information Design International Conference | CIDI 2015 [Blucher Design Proceedings, num.2, vol.2]. São Paulo: Blucher, 2015. ISSN 2318-6968, DOI 10.5151/designpro-CIDI2015-cidi_140 
construtivo; ambiental; de acessibilidade e segurança e normativo. De fato, ao longo do desenvolvimento de um projeto de sinalização são necessários conhecimentos e técnicas que perpassam as áreas de design gráfico, design de produto, design da informação, comunicação e arquitetura. Subjacente a todas essas áreas está a necessidade de comunicação: o papel fundamental da sinalização é comunicar algo a alguém. Essa comunicação, em geral, relaciona-se com o ambiente construído ou natural.

Como em todo projeto de design, para a realização de um projeto de sinalização é necessário realizar um levantamento de dados que esclareça as necessidades de comunicação a serem resolvidas. Neste artigo descrevemos o desenvolvimento da sinalização do Centro Universitário Norte do Espírito Santo, o campus da Universidade Federal do Espírito Santo localizado em São Mateus (ES), destacando os métodos e técnicas utilizados, dando ênfase especial às etapas de levantamento e sistematização dos dados.

Ao longo do processo foram elaboradas algumas ferramentas para aferir a pertinência das escolhas realizadas, no que se refere às informações a serem dispostas nos suportes da sinalização, bem como sua localização no espaço físico do campus. Dentre estas ferramentas, destacamos neste artigo os mapas de fluxos propostos e a maquete do campus contendo os elementos direcionais do sistema de sinalização. A principal contribuição dessas ferramentas foi possibilitar a aferição do funcionamento do sistema sem a necessidade de deslocamento até o Centro Universitário, distante $214 \mathrm{~km}$ do laboratório onde se localizava a equipe que desenvolveu o projeto.

A seguir descrevemos o contexto do projeto, bem como a metodologia utilizada e as ferramentas propostas.

\section{Contexto do projeto}

O projeto de sinalização do qual este artigo trata surgiu no âmbito do ProDesign Ufes, laboratório de projetos em design vinculado ao Departamento de Desenho Industrial da Universidade Federal do Espírito Santo. Atualmente o laboratório conta com dois coordenadores, professores do Departamento de Desenho Industrial, e quinze alunos do curso de design. O laboratório surgiu como um programa de extensão universitária, a partir da demanda da administração central da Universidade, no sentido de desenvolver projetos de sinalização para os seus quatro campi: Goiabeiras e Maruípe, localizados em Vitória e São Mateus e Alegre, localizados no interior do estado. Neste artigo tratamos especificamente do campus localizado na cidade de São Mateus, região Norte do estado. Ele também é conhecido como Centro Universitário Norte do Espírito Santo - Ceunes.

\section{O Centro Universitário Norte do Espírito Santo}

O atual campus do Ceunes começou a ser construído em 2005, às margens da BR-101. Sua construção foi planejada prevendo a sua expansão. Isso facilita o surgimento de novos prédios e mantém um certo padrão nas edificações, tanto em relação à arquitetura, quanto às distâncias entre os espaços construídos. Também devido ao planejamento, as vias de acesso para pedestres são largas e retas, dividindo o campus em quadras. Isso torna possível o acesso à maioria dos prédios, tanto por um vão central, quanto por suas laterais. Além disso, preenche o espaço com cruzamentos, criando múltiplas possibilidades de caminhos a serem traçados para se chegar a um destino. 
Figura 1: Planta arquitetônica do Ceunes.

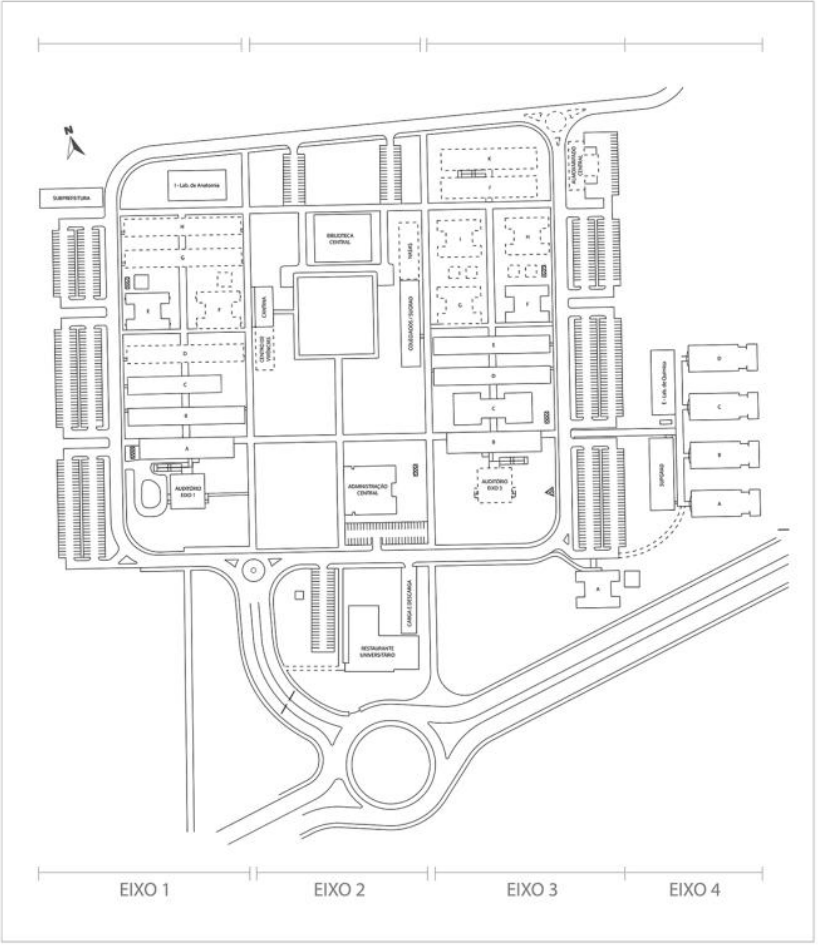

Em relação à distribuição dos edifícios no espaço, o campus é dividido em 4 setores que concentram serviços, ou cursos de áreas comuns. Esses setores foram nomeados pela administração como Eixos, e numerados conforme a sua localização no campus: os Eixos 1 e 3 concentram cursos de graduação; o Eixo 2 está relacionado à administração e serviços e o Eixo 4 abriga os cursos de pós-graduação.

Figura 2: Delimitação dos setores do campus, chamados oficialmente de "eixos" pela administração.

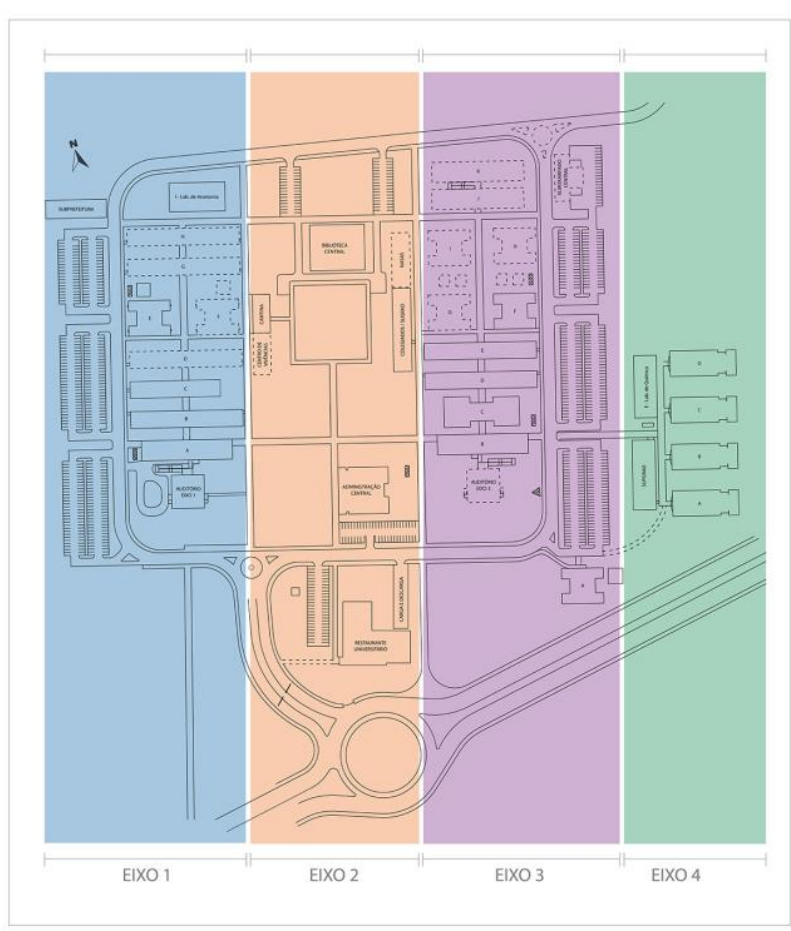


A maioria das edificações localizadas nos Eixos 1, 3 e 4 não pertencem a cursos específicos, sendo diferenciadas apenas por sua função: salas de aula, salas de professores ou laboratórios. A criação de novos cursos e a constante expansão do campus torna frequente a divisão e criação de novos departamentos, bem como mudanças na sua localização. Tendo em vista essas características, foi proposto também, pela própria administração do campus, que a identificação dos prédios fosse feita por um sistema alfanumérico, composto de blocos e eixos, como por exemplo: Bloco B - Eixo 3. Desse modo, como as nomenclaturas das edificações serão independentes das unidades administrativas, garante-se uma certa estabilidade de nomenclatura ao longo do tempo, tendo em vista as mudanças inevitáveis no futuro.

Tanto o sistema de eixos como a nomenclatura foram definidos oficialmente pela Universidade, porém o campus é relativamente novo e não possui nenhuma sinalização. Desse modo, as nomenclaturas oficiais adotadas ainda não são amplamente conhecidas pelos alunos, funcionários e frequentadores externos do local, abrindo espaço para a possibilidade de construção de uma identidade a partir da implementação do sistema.

\section{Metodologia}

O laboratório utiliza as metodologias de Follis e Hammer (1979) e de Calori (2007) como principais referências teóricas, mas sua aplicação se dá de maneira relativamente flexível, devido à situação particular do projeto e aos problemas concretos que foram sendo encontrados ao longo do processo. Follis e Hammer (1979) indicam quatro grandes fases no desenvolvimento de projetos dessa natureza:

1. Planejamento, que inclui as sub-etapas de levantamento de dados, análise de requisitos básicos, planejamento e desenhos esquemáticos;

2. Design, onde as peças de informação são efetivamente projetadas em sua integridade;

3. Documentação e orçamento, quando todas as especificações técnicas são definidas e documentadas para os fornecedores;

4. Supervisão, que inclui o acompanhamento da fabricação e instalação das peças.

Aqui, embora façamos menção a alguns elementos do design e dos materiais empregados, nosso foco de interesse se concentra na fase 1 , referente às diferentes etapas de planejamento. Na prática, observamos que essas fases não são um processo necessariamente linear, mas apenas categorias de problemas a serem resolvidos durante o projeto.

\section{Desenvolvimento}

No início do projeto o laboratório contava com poucas informações sobre o Ceunes: apenas um documento que descrevia o sistema de eixos e blocos e algumas plantas arquitetônicas desatualizadas. Convém destacar que o laboratório do ProDesign Ufes localiza-se no campus de Goiabeiras, na cidade de Vitória, a uma distância considerável do Ceunes - cerca de 214 $\mathrm{km}$, três horas e meia de carro. Com a necessidade de uma coleta de dados mais específica, foram realizadas visitas ao local de projeto e reuniões com a administração do campus. Nesses momentos foi possível observar o fluxo de circulação, identificar caminhos alternativos, conferir e atualizar a planta arquitetônica, mapear as entradas dos edifícios, aplicar questionários com usuários, testar protótipos e fazer registros fotográficos. Tendo em vista a dificuldade de acesso imediato ao local de projeto, as informações foram documentadas com rigor e detalhe, de modo que pudessem ser compartilhadas com a parte da equipe que não esteve presente in loco.

No laboratório, os dados coletados foram sistematizados e deram origem a novos documentos, como o mapa de fluxo de circulação. Esse último destaca os principais caminhos utilizados por pedestres e veículos e sinaliza a existência de caminhos alternativos. 
Figura 3: Mapa de fluxo de circulação. As linhas violeta indicam a circulação de veículos, enquanto as roxas mostram as rotas primárias de circulação de pedestres. As linhas em azul claro indicam rotas secundárias de pedestres, enquanto as verdes mostram alguns atalhos observados, não previstos inicialmente no plano diretor físico.

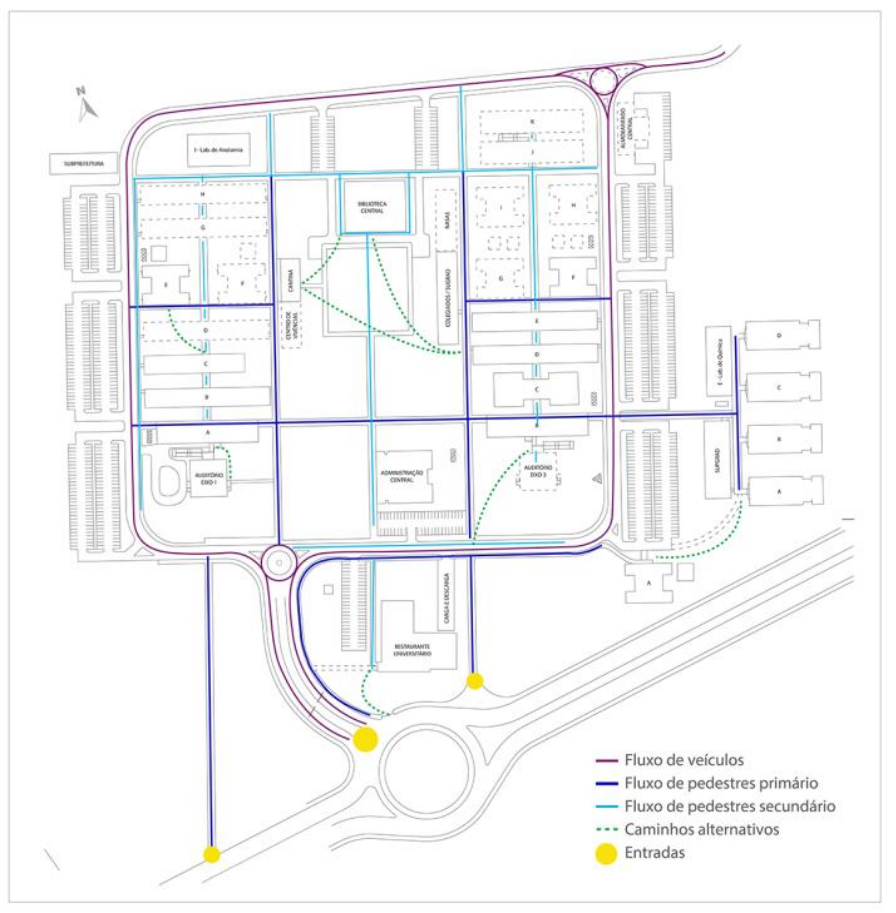

Com a sistematização dos dados, constatamos as diferentes necessidades de sinalização do campus, tornando possível, assim, o desenvolvimento dos elementos que seriam adequados para cada situação. Esses elementos são descritos a seguir:

Elementos identificadores do campus: o campus será identificado com um marco arquitetônico / escultura que possa ser vista da rodovia, paralela ao local. Serão também sinalizados seus portões de entrada, para que os pontos de acesso ao campus sejam facilmente identificados quando o usuário sair da via expressa.

Elementos identificadores das edificações: Os blocos serão identificados com totens externos de 4 faces. Os prédios que abrigam serviços principais serão identificados com grandes letras moldadas, para leitura à grande distância e os demais prédios serão identificados com lâminas de aço fixadas na fachada.

Placas direcionais: Considerando não somente a implantação do sistema, mas também sua manutenção ao longo do tempo, foram projetadas placas com estrutura modular. Desse modo, será possível a troca e inserção de apenas uma ou mais faixas de informação, sem a necessidade de substituir a estrutura inteira no momento de uma eventual atualização do sistema.

As placas Direcionais serão aplicadas em todos os cruzamentos das passarelas e a maior parte delas terá sua informação em duas faces: frente e verso. As informações contidas em cada placa serão agrupadas por eixos. Quanto às informações, só serão mostradas detalhadamente as direções de prédios de eixos próximos ao pedestre. Os eixos afastados terão apenas a direção do próprio setor informada na placa. Exemplificando: uma placa na passarela entre o Eixo 1 e o Eixo 2 informa as direções de todos os prédios dos Eixos 1 e 2. Já os prédios do Eixo 3 e 4 não aparecem, são apenas apresentadas as direções do eixo como um todo. Assim, na medida em que o usuário se aproxima do eixo de destino, seus conteúdos específicos vão sendo progressivamente revelados, evitando sobrecarga de informação. 
Figura 4: Exemplo de placa direcional de pedestres localizada entre os Eixos 2 e 3.

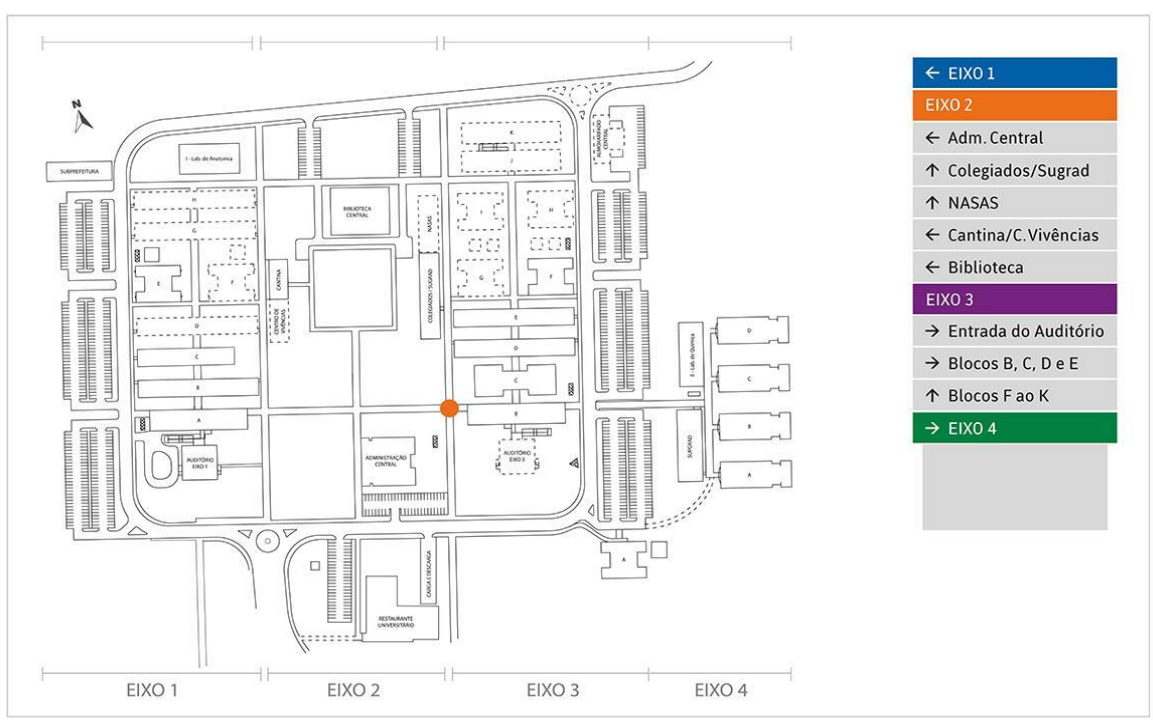

Diretórios: Para facilitar a compreensão de todo o espaço físico do Centro Universitário, bem como de seu sistema de eixos, serão instalados diretórios. Eles informam resumidamente o que está presente em cada eixo e apresentam um mapa do tipo "você está aqui", com informações detalhadas sobre o campus.

Cada eixo estará associado a uma cor, que estarão presentes nas placas identificadoras de edificações e serão reforçadas nas placas direcionais.

Durante a definição da tipologia dos elementos do sistema, foram criados códigos para os mesmos. Para a documentação dos dados, as placas foram agrupadas em um mapa, que marca a localização aproximada de cada uma. Também foi criada uma tabela para especificar os conteúdos de cada placa.

Figura 5: Mapa do plano de sinalização - indica a localização aproximada de cada peça do sistema. Estas são divididas entre placas direcionais, placas identificadoras e diretórios.

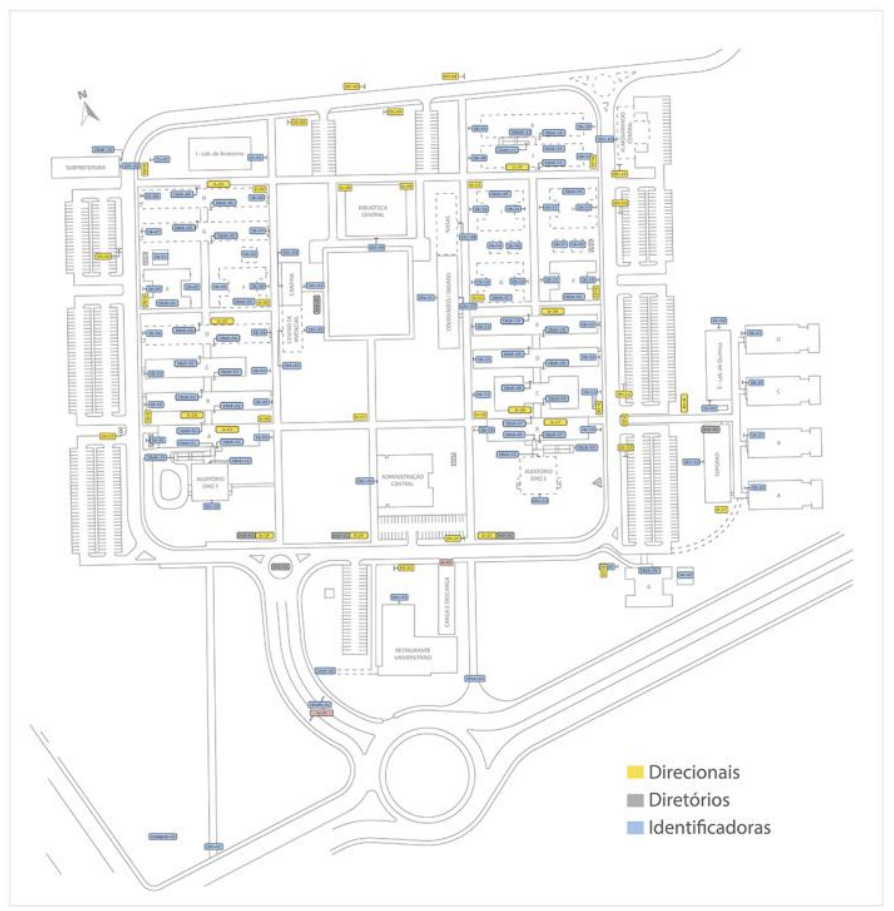


Figura 6: Tabela do plano de sinalização - contém os conteúdos e especificações de cada peça do sistema.

\begin{tabular}{|c|c|l|c|c|}
\hline código & número & \multicolumn{1}{|c|}{ conteúdo frente } & conteúdo verso & quantidade \\
\hline $\mathrm{IPr}$ & 1 & Auditório - EIXO 1 & & 1 \\
\hline $\mathrm{IPr}$ & 2 & Subprefeitura & & 1 \\
\hline $\mathrm{IPr}$ & 3 & Restaurante Universitário & & 2 \\
\hline $\mathrm{IPr}$ & 4 & Administração Central & & 1 \\
\hline $\mathrm{IPr}$ & 5 & Centro de Vivências & & 2 \\
\hline $\mathrm{IPr}$ & 6 & Cantina & & 2 \\
\hline $\mathrm{IPr}$ & 7 & Colegiados/Sugrad - Secretaria Única de Graduação & 2 \\
\hline $\mathrm{IPr}$ & 8 & NASAS - Núcleo de Assistência à Saúde do Servidor & & 1 \\
\hline $\mathrm{IPr}$ & 9 & Biblioteca & & 1 \\
\hline $\mathrm{IPr}$ & 10 & Auditório - EIXO 3 & & 1 \\
\hline $\mathrm{IPr}$ & 11 & Supgrad - Secretaria Única de Pós-Graduação & \\
\hline $\mathrm{IPr}$ & 12 & Almoxarifado central & & 2 \\
\hline $\mathrm{IPr}$ & 13 & Administração & & 1 \\
\hline $\mathrm{IPr}$ & 15 & Laboratório de Hidráulica & & 1 \\
\hline $\mathrm{IPr}$ & 16 & Laboratório de Mecanização Agrícola & & 1 \\
\hline $\mathrm{IPr}$ & 17 & Galpão de Oliricultura & & 1 \\
\hline $\mathrm{IPr}$ & 18 & Galpão de Viveiro & & 1 \\
\hline $\mathrm{IPr}$ & 19 & Galpão de Cafeicultura & & 1 \\
\hline $\mathrm{IPr}$ & 20 & Galpão de Maquinário & & 1 \\
\hline
\end{tabular}

Nesta etapa era necessário confirmar os locais das placas direcionais e seus conteúdos, bem como assegurar a boa navegabilidade e a coerência do sistema. Porém, a forma de documentação (mapa e tabela) deixava muito abstrata a visualização das informações, tornando difícil avaliar o sistema proposto.

\section{Novas Ferramentas: Maquete e Mapas de Fluxos Propostos}

Para resolver o problema da visualização das informações, a equipe do ProDesign Ufes criou uma maquete do campus. A planta baixa do Centro Universitário foi colada em uma placa isopor e as placas direcionais foram impressas e fixadas verticalmente no mapa com palitos de madeira. A escala utilizada na planta, 1:700, permitiu a manipulação confortável da maquete sobre a mesa de trabalho. As miniaturas de placas, entretanto, não seguiram sua proporção, tendo em vista que ficariam pequenas demais para a leitura das informações. Desse modo, foi utilizado um tamanho ampliado em relação ao mapa, de modo a tornar possível a navegação e permitir anotações de correções a serem feitas.

Figura 7: Maquete.

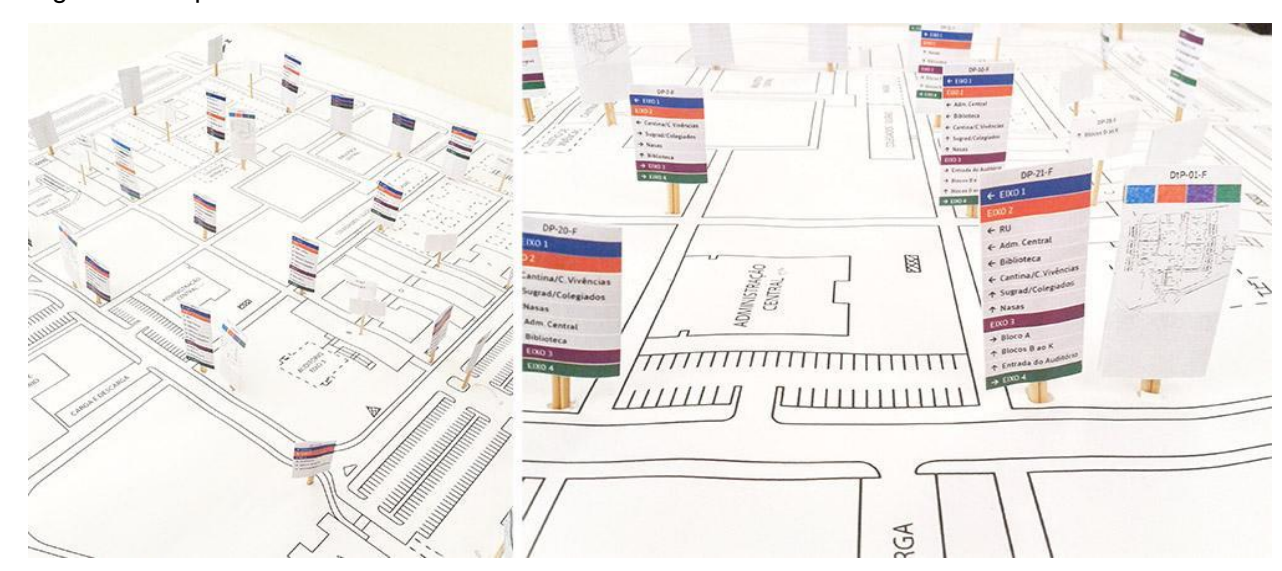

Anais [Pôster] do 7ํㅡㄹ Congresso Internacional de Design da Informação | CIDI 2015

Proceedings [Poster] of the 7th Information Design International Conference / IDIC 2015 
A tridimensionalidade proporcionada pela maquete facilitou a visualização das informações e a navegabilidade no sistema. Com ela foi possível confirmar a localização das placas: em qual lado da passarela ficariam e sua orientação (paralela ou perpendicular ao caminho). Além disso, serviu para simular futuros trajetos feitos pelos pedestres, o que permitiu perceber que algumas informações anotadas originalmente não asseguravam a coerência do sistema. Por exemplo, em alguns casos, ao ser guiado pelas placas, o pedestre caminharia em zigue-zague pelo campus, ao invés de percorrer um caminho mais direto.

Para suprir a necessidade de conferir a eficiência dos caminhos projetados, surgiu então uma outra ferramenta: os Mapas de Fluxos Propostos. Foi criado um mapa para cada prédio do campus e neles demarcados os locais de cada placa direcional. A partir de então, eram inseridas setas entre as placas, indicando a direção informada naquele ponto. Com esse método foi possível visualizar todo o caminho sugerido para se chegar a um prédio específico, bem como confirmar se estavam sendo utilizadas as possibilidades de caminhos mais objetivos.

Figura 8: Mapas de Fluxos Propostos - As setas longas indicam que a placa direciona o pedestre para a placa seguinte. As setas curtas indicam que após aquele ponto o pedestre já verá a placa identificadora da edificação.
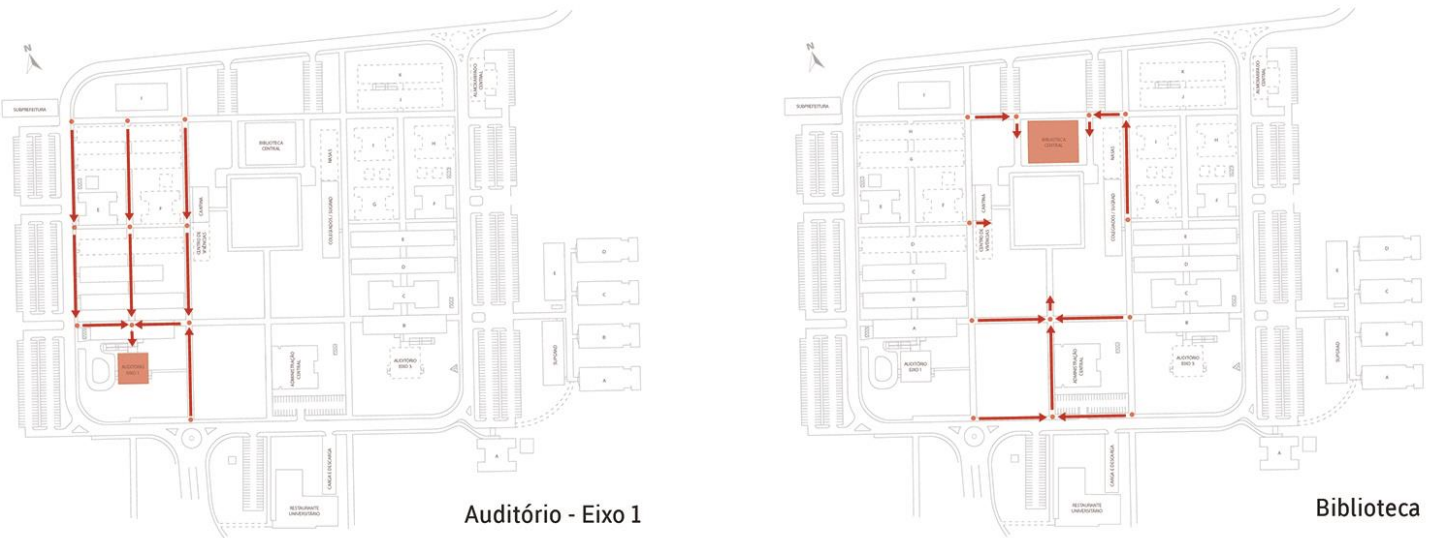

As duas ferramentas foram utilizadas em conjunto, até a definição final das informações. Os dois procedimentos auxiliaram a conferir a coerência do sistema e a assegurar sua boa navegabilidade. Especificamente, a maquete facilitou o ajuste na localização de cada placa no campus e os Mapas de Fluxos Propostos auxiliaram na definição dos conteúdos e direções.

\section{Conclusão}

A necessidade de desenvolver um projeto de sinalização para um local de difícil acesso promoveu a criação de novos métodos e ferramentas de análise. O método criado se mostrou eficaz para a realidade do Ceunes, um campus relativamente recente, com um traçado que facilita o entendimento do espaço. A metodologia utilizada pela equipe do ProDesign Ufes será replicada no desenvolvimento da sinalização dos demais campi da Universidade.

Atualmente, as Maquetes e os Mapas de Fluxos Propostos já estão sendo utilizados no projeto de sinalização do campus de Goiabeiras, em Vitória, cuja complexidade arquitetônicourbanística é significativamente maior que a do Campus de São Mateus, fruto de um crescimento mais orgânico ao longo dos 65 anos de sua existência.

Além disso, extrapolando a equipe do ProDesign Ufes, parte da metodologia está sendo utilizada no projeto da nova sinalização turística para pedestres da cidade do Rio de Janeiro. A equipe da empresa ICON, responsável pelo projeto, tem adotado a ferramenta dos Mapas como forma de facilitar o processo de seleção das informações a serem apresentadas nos elementos direcionais do sistema lá investigado.

Com estas duas experiências subsequentes, há indícios de que as Maquetes e, em especial, os Mapas do Fluxos Propostos, também podem ser pertinentes no planejamento de sistemas desenvolvidos para contextos mais complexos e amplos. 
No campo do design, a sinalização ainda é um tema que carece de literatura especializada no país, em especial no que se refere aos métodos e técnicas utilizados. Esperamos que este artigo some-se às referências sobre o assunto, e que estimule o debate entre os designers que trabalham com projetos de sinalização.

\section{Referências}

ADG Brasil. 2004. Guia ADG. São Paulo: Associação dos Designers Gráficos do Brasil.

CALORI, Chris. 2007. Signage and Wayfinding Design - A Complete Guide to Creating Environmental Graphic Design Systems. Hoboken, New Jersey: John Wiley \& Sons.

COSTA, Joan. 2007. Señaletica Corporativa. Barcelona: Editora do autor.

FOLLIS, John; HAMMER, Dave. 1979. Architectural Signing and Graphics. London: The Architectural Press.

PASSINI, Romedi; ARTHUR, Paul. 2002. Wayfinding: People, Signs, and Architecture. Ontario:

Focus Strategic Communications Incorporates.

VELHO, Ana Lucia de Oliveira Leite. MAGALHÃES, Cláudio de Freitas (Orientador). 2007. O

Design de Sinalização no Brasil: a introdução de novos conceitos de 1970 a 2000.

Dissertação de Mestrado. Departamento de Artes e Design. Pontifícia Universidade Católica do Rio de Janeiro, Rio de Janeiro.

\section{Sobre os autores}

Mauro Pinheiro, Dr., Ufes / Esdi-Uerj, Brazil <mauro.pinheiro@gmail.com>

Ricardo Esteves, MSc., Ufes, Brazil <ricardo@outrasfontes.com>

Viviani Carnielli, Graduanda, Ufes, Brazil <viviani.gc@gmail.com> 